\title{
APLICAÇÃO DA FUNÇÃo HIPERBóLICA NA CONSTRUÇÃO DE CURVAS DE ÍNDICE DE LOCAL ${ }^{1}$
}

\begin{abstract}
Daniel Henrique Breda Binoti ${ }^{2}$, Mayra Luiza Marques da Silva Binoti ${ }^{3}$ e Hélio Garcia Leite ${ }^{4}$
RESUMO - Neste estudo foi avaliada a eficiência da função hiperbólica, para geração de curvas anamórficas e polimórficas de índices de local. As curvas de índice de local foram construídas utilizando os métodos: da curva-guia, da equação das diferenças, da predição de parâmetros, de Hammer e dos índices de local definidos preliminarmente. Para aplicação desses métodos foram utilizados dados de parcelas permanentes de povoamentos de eucalipto. A eficiência foi confirmada, sendo a função indicada para descrever tendências polimórficas e anamórficas, podendo ser utilizada na classificação da capacidade produtiva de povoamentos de eucalipto.
\end{abstract}

Palavras-chave: Índice de sítio, Função Hiperbólica e Eucalipto

\section{APPLICATION OF HYPERBOLIC FUNCTION CURVES IN CONSTRUCTION SITE INDEX}

\begin{abstract}
This study evaluated the efficiency of the hyperbolic function for construction of anamorphic and polymorphic site index curves. The site index curves were constructed using the methods: guide-curve, difference equation, parameter prediction, Hammer and site index preliminarily defined. To apply these methods data from permanent plots in stands of eucalyptus were used. The efficiency was confirmed, being the function indicated to describe anamorphic and polymorphic trends that can be used in the classification of productivity capacity of eucalypt stands.
\end{abstract}

Keywords: Site index, Hyperbolic function and Eucalypt.

\section{INTRODUÇÃ̃O}

A classificação da capacidade produtiva pode ser feita por métodos diretos ou por métodos indiretos. Na classificação direta são construídas curvas de índices de local, sendo esses índices iguais às alturas dominantes $(H d)$ em uma idade de referência, ou idade-índice. A altura total das árvores dominantes é um bom indicador da capacidade produtiva, conforme apontado por Hann e Scrivani (1987) e Campos e Leite (2009), entre outros.
Pesquisas sobre índices e qualidade de local são relativamente antigas, por exemplo: Roth (1916), Spring (1917), Frothingham (1918), Bull (1931), Meyer (1940), Husch (1956) e Vincent (1961). A partir da década de 1960, inúmeros trabalhos têm sido encontrados na literatura, na maioria das vezes apresentando curvas de índices de local para determinada espécie e região (DIAS et al., 2005; CRUZ et al., 2008).

\footnotetext{
${ }^{1}$ Recebido em 12.08.2010 e aceito para publicação em 28.05.2012.

2 Programa de Pós-Graduação em Mestrado em Doutorado em Ciência Florestal. Universidade Federal de Viçosa, Departamento de Engenharia florestal. E-mail: <danielhbbinoti@gmail.com>.

${ }^{3}$ Universidade Federal dos Vales do Jequitinhonha e Mucuri, Departamento de Engenharia Florestal.E-mail:<MayraBinoti@gmail.com>.

${ }^{4}$ Universidade Federal de Viçosa, Departamento de Engenharia florestal. E-mail <hgleite@ gmail.com>.
} 
Curvas de índice de local podem ser do tipo anamórfico, polimórfico ou polimórfico disjunto, dependendo dos métodos de análise empregados e das procedências dos dados (CLUTTER et al., 1983). No Brasil tem predominado o emprego de curvas anamórficas e, entre os métodos de construção, tem sido mais comum o emprego do método da curva-guia, conforme descrito em Campos e Leite (2009).

Para a construção de curvas de índices de local, é necessário definir um modelo que represente a relação da altura com a idade, bem como definir o comportamento da família de curvas geradas por esse modelo, que deve ser flexível para gerar curvas com padrão exponencial ou sigmoidal. Isso porque, na maioria das vezes, a relação $H d$ vs. Idade ( $I$ ) segue um desses padrões. Conforme Campos e Leite (2009), para plantios de Eucalyptus, Pinus e Teca no Brasil tem sido comum o uso do método da curva-guia com o emprego da relação funcional $\mathrm{Ln} H d$ $=f\left(I^{-1}\right)$, ou das funções logística ou Chapman-Richards. No primeiro caso, a relação implícita entre $H d$ e idade é exponencial e nos outros dois, sigmoidal.

Até 2010 não foi encontrado na literatura o uso da função hiperbólica para construção de curvas de índices de local. Essa função hiperbólica foi proposta por Guimarães (2002), para descrição de distribuições de diâmetro em povoamentos equiâneos e se mostrou bastante flexível para descrição de diferentes formas de curvas, indicando a possibilidade de uso para a construção de curvas de índices de local, anamórficas ou polimórficas.

O objetivo deste estudo foi avaliar a eficiência da função hiperbólica (GUIMARÃES, 2002) para a construção de curvas anamórficas e polimórficas de índices de local.

\section{MATERIAL E MÉTODOS}

\section{Descrição dos dados}

Foram utilizados os dados de altura dominante e idade publicados por Campos e Leite (2009), obtidos de 20 parcelas permanentes instaladas em um povoamento de eucalipto e medidas nas idades de 36 , 48, 60, 72 e 84 meses (Tabela 1 ).

\section{Modelo estatístico e expressão analítica dos índices de local}

A função hiperbólica foi descrita por Guimarães (2002) e pode ser expressa da seguinte forma:

$$
H D=\alpha_{1}\left(\tanh \left(\alpha_{2}\left(I-\alpha_{3}\right)^{\alpha_{4}}\right)+e\right.
$$

em que:

$H d=$ altura média das árvores dominantes, em m;

$I=$ idade dos povoamentos, em meses;

$\alpha_{1}, \alpha_{2}, \alpha_{3}$, e $\alpha_{14}=$ parâmetros dos modelos;

tanh $=$ tangente hiperbólica; e

e = erro aleatório, e $\sim \mathrm{N}(0$, ó $)$.

Para a construção das curvas de índice de local foi utilizada uma idade-índice de 72 meses e os métodos da curva-guia (2), da equação das diferenças (3), da predição de parâmetros (4), de Hammer (5) e parâmetros definidos preliminarmente (6):

$$
\begin{aligned}
& H d=\frac{S\left(\tanh \left(\alpha_{1}\left(I-\alpha_{2}\right)^{\alpha_{3}}\right)\right.}{\tanh \left(\alpha_{1}\left(I \alpha_{3} \alpha_{3}\right)\right.}+e \\
& H d_{2}=\frac{\left.H d_{1}^{\tanh \left(\operatorname { t a n h } \left(\alpha_{1}\left(\alpha_{1}\left(I_{2}-\alpha_{2} \alpha_{2}\right)^{\alpha_{3}}\right)_{3}\right.\right.}\right)}{\tanh \left(\alpha_{1}\left(I_{1}-\alpha_{2}\right)^{\alpha_{3}}\right)}+e \\
& H d=\beta_{0}\left(\tanh \left(\beta_{1}\left(I-\beta_{2}\right)^{\beta_{3}}\right)+e\right. \\
& H d=\left(\gamma_{0}+\gamma_{1} S\right) \tanh \left(\alpha_{1}\left(I-\alpha_{2}\right)^{\alpha_{3}}\right)+e \\
& H d=\alpha_{0} S \tanh \left(\alpha_{1}\left(I-\alpha_{2}\right)^{\alpha_{3}}\right)+e
\end{aligned}
$$

em que:

$\beta_{0}=\alpha_{0}+\alpha_{1} S+e$

$\beta_{1}=\frac{\alpha_{0}}{\left(1-\alpha_{1} e^{\left(-\alpha_{2} \mathrm{~S}\right)}\right)}+e$

$\beta_{2}=\alpha_{0}+\alpha_{1} S+\alpha_{2} S^{2}+e$

$\beta_{3}=\alpha_{1}+\alpha_{2} S$

$H d=$ altura média das árvores dominantes, em m;

$I=$ idade dos povoamentos, em meses;

$\alpha_{1}, \alpha_{2}, \alpha_{3}, \alpha_{4}, \gamma_{1}, \gamma_{2}=$ parâmetros dos modelos;

tanh $=$ tangente hiperbólica; $\mathrm{e}$

$\mathrm{e}=$ erro aleatório, $\mathrm{e} \sim \mathrm{N}(0, \sigma 2)$

\section{Ajuste e avaliação}

Os modelos foram ajustados pelo método de mínimos quadrados não lineares, empregando o procedimento iterativo Quasi-Newton e o software Statistica. 
Os modelos foram avaliados individualmente com base em coeficiente de correlação entre valores observados e estimados de altura dominante, análise gráfica da relação entre as alturas dominantes observadas e a estimadas, análise gráfica de resíduos e consistência entre as curvas de índices de local e a amplitude de alturas dominantes observadas.

\section{RESULTADOS}

Todos os modelos testados tiveram ajustes considerados como satisfatórios, com estimativas de correlação entre alturas observadas e alturas estimadas superiores a 95\%. Essas correlações, bem como as análises de resíduos, não foram apresentadas, embora tenham sido utilizadas para a tomada de decisão. Para todos os métodos de construção das curvas de índices de local, foram estabelecidas três classes de local, sendo obtidas curvas consistentes com a amplitude de alturas dominantes observadas (Figura 1).

A equação estimada para a construção das curvas de índices de local pelo método da curva-guia foi:

$$
H d=28,7506 \tanh \left(0,0045(I-0,6818)^{1,3497}\right)
$$

e pelo método da equação das diferenças,

$$
H d=1,1397 \tanh \left(0,0060(I-2,6535)^{1,2743}\right)
$$

Visando à construção de curvas polimórficas, pelo método da predição de parâmetros a função hiperbólica foi ajustada aos dados de cada parcela. As equações ajustadas foram aplicadas para estimar a altura dominante na idade índice(S). Em seguida, foram testados diferentes modelos relacionando os parâmetros da função hiperbólica com os índices de local, sendo selecionadas as seguintes equações:

$$
\begin{gathered}
\alpha_{0}=-13,2168+1,6620 S \\
\alpha_{1}=\frac{0,0039}{\left(1-22,6020 e^{(-0,2015 \mathrm{~S})}\right)} \\
\alpha_{2}=-75,5017+6,5176 S-0,1304 \mathrm{~S}^{2} \\
\alpha_{2}=1,6498-0,0106 \mathrm{~S}
\end{gathered}
$$

Substituindo essas equações na função (1), o resultado foi:

$$
\begin{aligned}
& H d=(-13,2168+1,6620 S) \tanh \left(\frac{0,0039}{\left.1-22,6020 e^{(-0,2015 S}\right)}\right. \\
& \left.\left(\left(I-\left(-75,5017+6,5176 S-0,1304 S^{2}\right)\right)^{1,6498-0,01065}\right)\right)
\end{aligned}
$$

Para a construção das curvas de indices de local pelos métodos de Hammer (14) e utilizando índices preliminares (15), foram geradas as seguintes expressões:

$\mathrm{Hd}=\left(3,552 e^{-15}+1,1154 S\right) \tanh \left(0,0044(I-0,4584)^{1,3581}\right)$

$H d=1,1397 \operatorname{Stanh}\left(0,0060(I-2,6535)^{1,2743}\right)$

As curvas anamórficas de índices de local obtidas pelos métodos da curva-guia, da equação das diferenças, de Hammer e dos índices de local definidos preliminarmente apresentaram características semelhantes (Figura 1). Nessa mesma figura foi apresentado o feixe polimórfico construído com o emprego do método da predição de parâmetros e as curvas geradas para o índice de local médio (altura dominante de $27 \mathrm{~m}$ aos 72 meses de idade), empregando-se cada um dos métodos de construção.

Para comprovar a flexibilidade da Função hiperbólica, foram empregados ainda dados de parcelas permanentes de oito clones com diferentes tendências de crescimento em altura dominante (Figura 2).

\section{DISCUSSÃO}

A classificação da capacidade produtiva em povoamentos de eucalipto no Brasil geralmente é realizada com o emprego do método da curva-guia (CAMPOS; LEITE, 2009). Contudo, esse método não permite a construção de curvas polimórficas, sendo diferentes tendências de crescimento não representadas pelos modelos. Por isso, funções que apresentem correlações entre seus parâmetros e os valores de sítio são fundamentais para a construção de curvas pelo método da predição de parâmetros.

A função também se mostrou eficiente para a descrição de diferentes tendências de crescimento (Figura 2), desde tendências exponenciais (A, B, C, D e H) a tendências assintóticas ( $\mathrm{E}, \mathrm{F}$ e $\mathrm{G}$ ), podendo esta ser usada para a classificação da capacidade produtiva de povoamentos de eucalipto no Brasil por todos os métodos testados neste trabalho.

Revista Árvore, Viçosa-MG, v.36, n.4, p.741-746, 2012 
Curva-guia
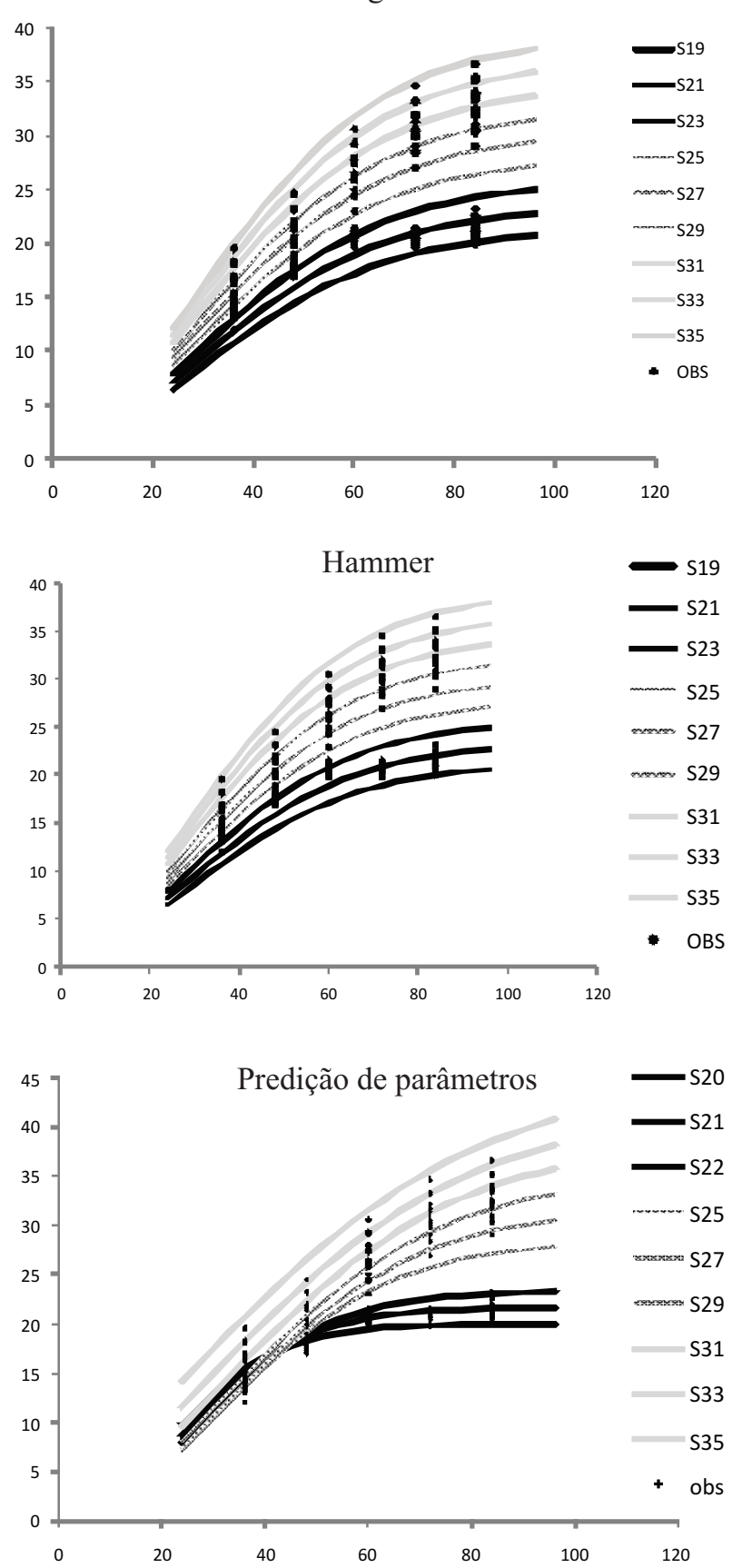

Equação das diferenças
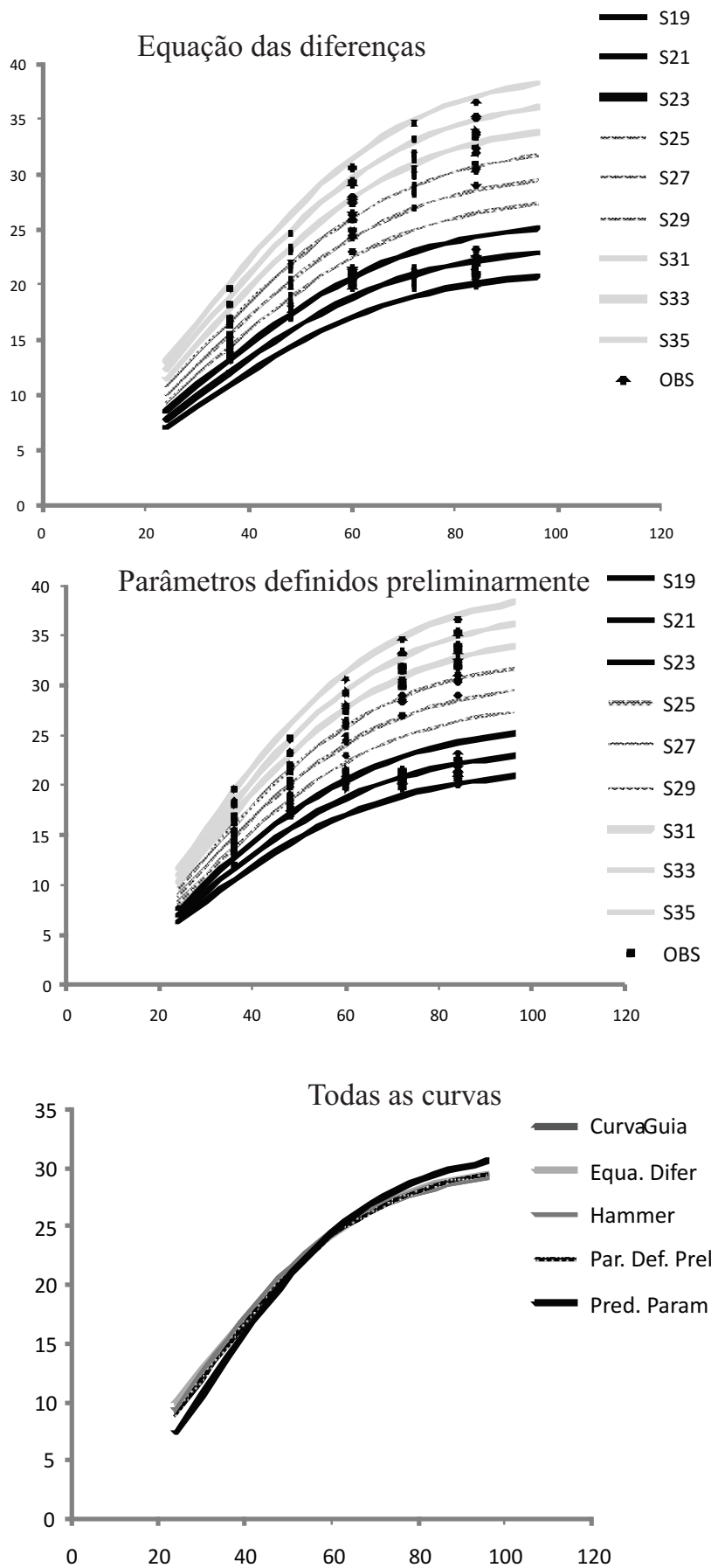

Figura 1 - Curvas de índices de local estimadas empregando diferentes métodos de construção, o modelo hiperbólico e uma idade-índice de 72 meses.

Figure 1 -Estimated site index curves estimated using different methods of construction, the hyperbolic model and index age of 72 months.

Revista Árvore, Viçosa-MG, v.36, n.4, p.741-746, 2012 

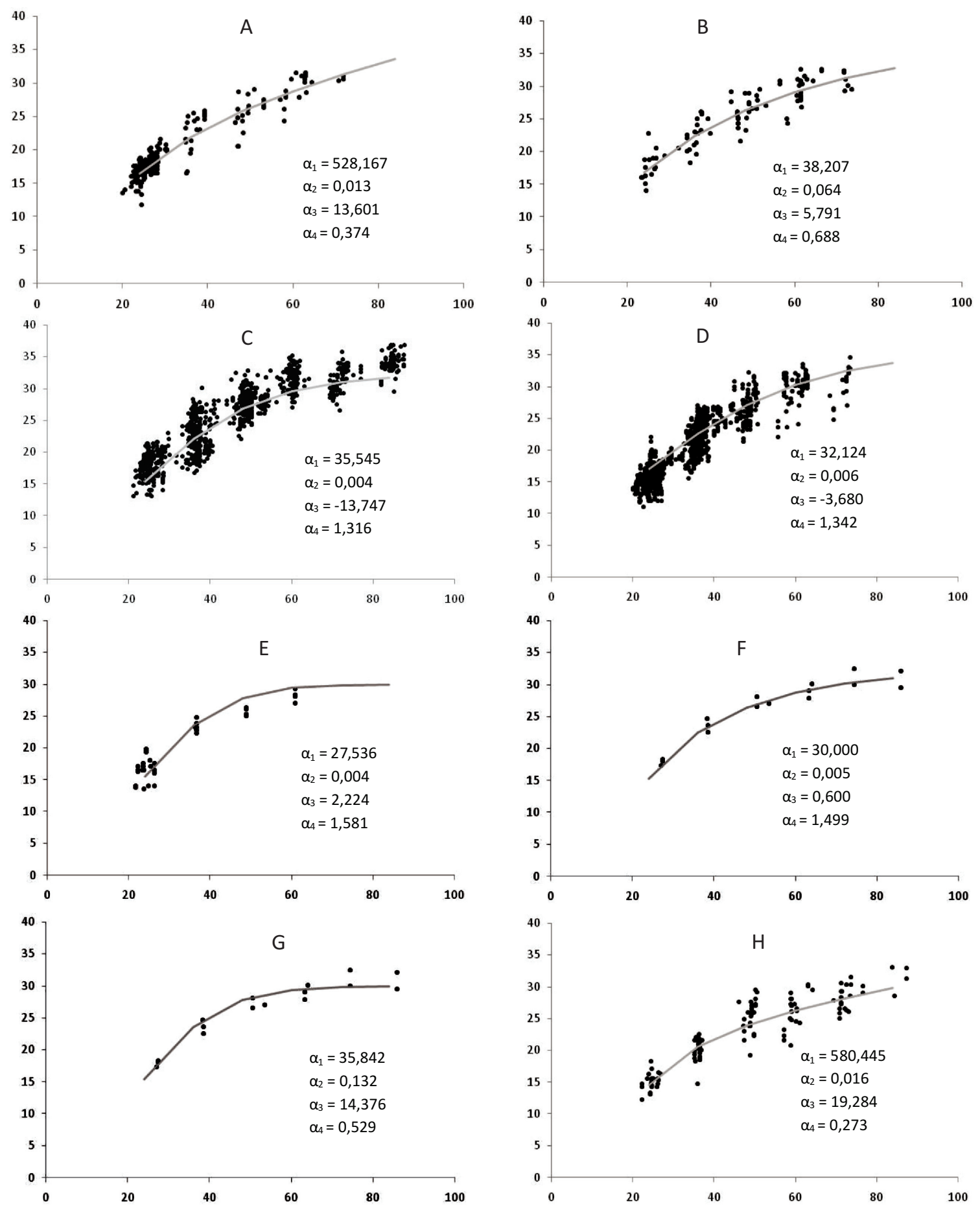

Figura 2 - Flexibilidade da função hiperbólica em expressar o crescimento em altura dominante para dados de parcelas permanentes de oito materiais genéticos distintos.

Figure 2 - Flexibility of the hyperbolic function to express the growth in dominant height for data of permanent plots of eight different genetic materials. 


\section{CONCLUSÕES}

A função hiperbólica mostrou flexibilidade e realismo biológico adequado, podendo ser utilizada para a classificação da capacidade produtiva de povoamentos equiâneos.

\section{REFERÊNCIAS}

BULL, H. The use of polymorphic curves in determining site quality in young red pine plantations. Journal of Agricultural Research, v.43, n.1 p.1-29, 1931.

CAMPOS, J. C. C.; LEITE, H. G. Mensuração florestal: perguntas e respostas. 3.ed. rev. e ampl. Viçosa, MG: Universidade Federal de Viçosa, 2009. 548p.

CLUTTER, J. L. et al. Timber management -A quantitative approach. New York: Wiley, 1983. 333p.

CRUZ, J. P. et al. Curvas de crescimento e de índice de local para povoamentos

de Tectona grandis em Tangará da Serra, Mato Grosso. Revista Árvore, v.32, n.4, p.679-685, 2008.

DIAS, A. N. et al. Avaliação de métodos de ajuste de curvas de índices de local em povoamentos de eucalipto desbastados. Revista Árvore, v.29, n.5 p.741-747, 2005.
FROTHINGHAM, E. H. Height growth as a key to site. Journal of Forestry, v. 16, n.17, p.754-760, 1918.

GUIMARÃES, D. P. Uma função

hiperbólica de distribuição probabilística de alta flexibilidade. Planaltina: Embrapa Cerrados, 2002. 40p.

HANN, D. W.; SCRIVANI, J. Dominant-heightgrowth and site-index equations for douglas-fir and ponderosa pine in Southwest Oregon.

Corvallis: Forest Research Laboratory, Oregon State University, 1987. (Research Bulletin, 59)

HUSCH, B. Use of age at D.B.H. as a variable in the site index concept. Journal of Forestry, v.54, n.5, p.340, 1956.

MEYER, H. A. A mathematical expression for height curves. Journal of Forestry, v.38, p.415-420, 1940 .

ROTH, F. Concerning site. Forestry Quarterly, v.3, n.1, p.3-13, 1916.

SPRING, S. N. Notes and comments: site and site classes. Journal of Forestry, v.15, p.102, 1917.

VINCENT, A. B. Is height/age a reliable index of site? Forestry Chronicle, v.37, n.2, p.144-150, 1961. 